\title{
Gênero e hidratação como mediadores relevantes para restauração de processos inflamatórios em atletas jovens
}

\section{Gender and hydration as relevant mediators for restoration of inflammatory processes in young athletes}

\section{Flávio ignácio bachini}

Doutor Neurologia - Universidade Federal do Estado do Rio de Janeiro (UNIRIO), Rio de Janeiro, Brasil.

Instituição: Comitê Olímpico Brasileiro (COB).

Endereço: Av. das Américas, 899 - Barra da Tijuca, Rio de Janeiro - RJ, 22631000.

E-mail: f.bachini@gmail.com

\section{Leonardo Macedo}

Especialista em Personal Trainer - Faculdade Venda Nova do Imigrante (FAVENI), Espirito Santo, Brasil.

Instituição: Prefeitura Municipal de Barra do Garças (PMBG), Barra do Garças, Brasil.

Endereço: R. Carajás, 522 - Centro, Barra do Garças - MT, 78600-000.

E-mail: leonardodossantos.bg@gmail.com

\section{Luis Carlos Oliveira Gonçalves}

Mestre em Ciência da Motricidade Humana - Universidade Castelo Branco (UCB), Rio de Janeiro, Brasil; Doutorando em Ciências da Saúde Universidade Federal de Mato Grosso (UFMT). Faculdade de medicina, Cuiabá, Brasil.

Instituição: Universidade Federal do Estado do Rio de Janeiro (UNIRIO), Laboratório de Bioquímica de Proteínas.

Endereço: Av. Pasteur, 296 - Urca. 22290-240 - Rio de Janeiro, RJ - Brasil

E-mail: luisogoncalves@yahoo.com.br

\section{Adenilda Cristina Honório França}

Doutora em Imunologia pela Universidade de São Paulo (USP), São Paulo, Brasil.

Instituição: Universidade Federal de Mato Grosso (UFMT), Barra do Garças, Brasil.

Endereço: Instituto de Ciências Biológicas e da Saúde. Rodovia BR 070, Km 5. 78600-000 - Barra do Garcas, MT - Brasil.

E-mail: adenilda.franca@ufmt.br

\section{Eduardo Luzia França}

Doutor em Imunologia pela Universidade de São Paulo (USP), São Paulo, Brasil. Instituição: Universidade Federal de Mato Grosso (UFMT), Barra do Garças, Brasil.

Endereço: Instituto de Ciências Biológicas e da Saúde. Rodovia BR 070, Km 5. 
78600-000 - Barra do Garcas, MT - Brasil.E-mail: dr.eduardo.franca@gmail.com

Aníbal Monteiro De Magalhães Neto

Doutor em Genética e Bioquímica - Universidade Federal de Uberlândia (UFU). Uberlândia, MG - Brasil.

Instituição: UFMT - Instituto de Ciências Biológicas e da Saúde.

Endereço: Rodovia MT 100, Km 3,5 s/n. Centro. 78696000 - Pontal do

Araguaia, MT - Brasil.

E-mail: professoranibal@yahoo.com.br

\section{RESUMO}

Objetivos: Poucos estudos tem dado a devida importância ao estado de hidratação e as alterações hematológicas em menores de 18 anos. O presente estudo investigou estas alterações em menores de 18 anos na prova de 3000 metros para ambos os gêneros. Método: Foram incluídos os seis melhores participantes das Olimpíadas Nacionais do Ensino Médio (menores de 18 anos) no Brasil. Trata-se de um estudo observacional e transversal. Os atletas correram 3.000 metros em uma pista oval de 400 metros. Foi realizado leucograma total e específico, eritrograma e contagem de plaquetas em quatro diferentes tempos (jejum, pré, pós e recuperação). Os cálculos de comparação entre tempos, apresentados, foram adaptados do modelo matemático de variação percentual. Resultados: Quando comparadas as médias em relação a cada tempo, pode-se notar que os eritrócitos, hemoglobina, hematócrito, leucócitos totais e basófilos aumentaram significativamente após a corrida para ambos os gêneros. Além disso, as plaquetas também aumentaram para ambos os gêneros sem significância. O coeficiente de correlação de Pearson mostrou importante interação leucócitos-plaquetas. Os homens recuperaram seu estado de hidratação em quase metade do tempo em relação as mulheres. Em relação ao sistema imune e resposta inflamatória, as atletas do gênero feminino tiveram um aumento relativo de mais que o dobro para leucócitos e não recuperaram estes valores, além de apresentarem um aumento de eosinófilos neste mesmo tempo, enquanto os atletas do gênero masculino apresentaram diminuição. As mulheres apresentaram um aumento de quase o dobro para linfócitos, enquanto os homens tiveram este comportamento para os fagócitos. Conclusões: $O$ exercício induz alteração do estado de hidratação e sistema imune, tendo diferenças em relação ao gênero. Quando bem conduzido, gera efeitos antiinflamatórios a longo prazo. Quando não há controle sobre o volume, intensidade e recuperação pode causar imunossupressão e promover a suceptibilidade para infecções. Foi possível observar que os atletas do gênero masculino tem um menor estresse inflamatório e recuperam o seu estado de hidratação e a resposta inflamatória mais rápido que as meninas após uma corrida de 3000 metros.

Palavras-chave: Sistema Imunológico, Hidratação, Hematologia, Atletas, Medicina Esportiva.

\section{ABSTRACT}

Objectives: Few studies have given due importance to the hydration status and 


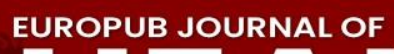 \\ Big HEALTH

hematological alterations in children under 18 years of age. The present study investigated these alterations in children under 18 years old in the 3000 meters test for both genders. Method: The six best participants of Brazil's National High School Olympiads (under 18 years old) were included. This is an observational and cross-sectional study. Athletes ran 3,000 meters on a 400 meters oval track. Total and specific white blood cell count, erythrogram, and platelet count were performed four times (fasting, pre, post, and recovery). The time comparison calculations presented were adapted from the percentage variation mathematical model. Results: When comparing the means for each time, it can be noted that erythrocytes, hemoglobin, hematocrit, total leukocytes, and basophils increased significantly after the race for both genders. Furthermore, platelets also increased for both genders without significance. Pearson's correlation coefficient showed a significant leukocyte-platelet interaction. Men regained their hydration status in almost half the time compared to women. Regarding the immune system and inflammatory response, female athletes had a relative increase of more than double for leukocytes and did not recover these values, in addition to showing an increase in eosinophils simultaneously, while male athletes showed a decrease. Women showed an increase of almost double for lymphocytes, while menshowed this behavior for phagocytes. Conclusions: Exercise induces changes in hydration status and immune system, with differences concerning gender. When done well, it generates long-term anti-inflammatory effects. When there isno control over volume, intensity, and recovery, it can cause immunosuppressionand increase infection susceptibility. It was observed that male athletes have lower inflammatory stress and recover their hydration status and inflammatory response faster than girls after a $\mathbf{3 0 0 0}$ meter run.

Keywords: Immune System, Hydration, Hematology, Athletes, Sports Medicine.

\section{RESUMEN}

Pocos estudios han dado la debida importancia al estado de hidratación y alteraciones hematológicas en niños menores de 18 años. El presente estudio investigó estas alteraciones en menores de 18 años en la prueba de 3000 metros para ambos sexos.

Método: Se incluyeron los seis mejores participantes de las Olimpíadas Nacionales de Bachillerato (menores de 18 años) en Brasil. Este es un estudio observacional y transversal. Los atletas corrieron 3.000 metros en una pista ovalada de 400 metros. El recuento total y específico de glóbulos blancos, el eritrograma y el recuento de plaquetas se realizaron en cuatro momentos diferentes (ayuno, pre, post y recuperación). Los cálculos de comparación de tiempo presentados fueron adaptados del modelo matemático de variación porcentual. Resultados: Al comparar las medias para cada tiempo, se puede observar que los eritrocitos, hemoglobina, hematocrito, leucocitos totales y 
basófilos aumentaron significativamente después de la carrera para ambos sexos. Además, las plaquetas también aumentaron para ambos sexos sin importancia. El coeficiente de correlación de Pearson mostró una importante interacción leucocitos-plaquetas. Los hombres recuperaron su estado de hidratación en casi la mitad del tiempo en comparación con las mujeres. En cuanto al sistema inmunológico y la respuesta inflamatoria, las deportistas presentaron un aumento relativo de más del doble para los leucocitos y no recuperaron estos valores, además de mostrar un aumento de eosinófilos al mismo tiempo, mientras que los deportistas masculinos mostraron una disminución. Las mujeres tuvieron un aumento de linfocitos casi el doble, mientras que los hombres tuvieron este comportamiento para los fagocitos. Conclusiones: El ejercicio induce cambios en el estado de hidratación y el sistema inmunológico, con diferencias en relación al sexo. Cuando se hace bien, tiene efectos antiinflamatorios a largo plazo. Cuando no hay control sobre el volumen, la intensidad y la recuperación, puede causar inmunosupresión y promover la susceptibilidad a las infecciones. Se pudo observar que los atletas masculinos tienen menor estrés inflamatorio y recuperan su estado de hidratación y respuesta inflamatoria más rápido que las niñas después de una carrera de 3000 metros.

Palabras Clave: Sistema inmunológico, Hidratación; Hematología, Atletas, Medicina deportiva.

\section{INTRODUÇÃO}

A água representa em torno de $60 \%$ do corpo humano (Liska et al., 2019). Homens e mulheres praticantes de exercícios e esportes não consomem as quantidades adequadas de água, sendo a ingestão por mulheres menor que a dos homens (Martin et al., 2019). O planejamento da ingestão de água com base na diferença entre os sexos, modalidade esportiva e outros aspectos como temperatura e umidade se faz necessária para a melhora da performance e manutenção da saúde de atletas e praticantes de exercícios físicos (Ayotte and Corcoran 2018). Adicionalmente, as respostas termorregulatórias ao estresse ambiental são semelhanete em meninas adolescentes e mulheres adultas, assim como, em meninos adolescentes e homens adultos (Rodrigues et al.,2019). (Leites et al., 2016).

A hipoidratação está associada ao desequilíbrio na homeostase corporal envolvendo processos metabólicos, a cognição, a habilidade técnica e o desempenho de atletas, o que pode causar perda no desempenho e prejuízos a 
saúde do indivíduo (Aristotelous et al., 2018; Nuccio et al., 2017). Comumente observado nos Jogos de Verão, a temperatura elevada e a hipoidratação resultam em alterações significativas na osmolaridade plasmática e volume plasmático com possíveis reflexos nas funções neurais e motoras (Vadopalas et al., 2019). É notório que a umidade elevada do clima tropical induz ao aumento de evaporação pelo suor, promove a termorregulação resultando em hipoidratação e redução do desempenho físico (Hermand et al., 2019; Muth et al., 2019). Os estados subclínicos e clínicos relacionados a hidratação frequentemente são desvalorizados pela comissão técnica e inadequadas estratégias são adotadas (Lacey et al., 2019)Malisova et al., 2016).

Os atletas de elite estão constantemente expostos a hipoidratação, distúrbios fisiológicos, neuroendócrinos e metabólicos, ao pobre estado nutricional, à sobrecarga de treinamento/competição, viagens e ao ambiente, todos fatores que podem culminar em imunossupressão e risco de infecção (Brolinson and Elliott 2007; Simpson et al., 2015). Por outro lado, o exercício induz mudanças no sistema imunológico que geram efeitos antiinflamatórios a longo prazo, a depender da sua intensidade e duração (Moro-Garcia et al., 2014). Por exemplo, o exercício físico moderado pode melhorar a imunidade e o intenso pode causar imunossupressão durante a recuperação que pode durar de 3 a 72 horas (Pedersen and Ullum, 1994).

(Nieman 2003). (Peake and Neubauer 2017; Schwellnus and Soligard 2016).

Intensidade/volume alto de exercícios, hipoidratação, assim como, à hipertermia induzem à alterações na cascata de coagulação e aparecimento de inflamação sistêmica (Pyne et al., 2014; Pedersen and Ullum, 1994; Vadopalas et al., 2019). O frio também pode alterar as condições metabólicas e circulatórias e deprimir o sistema imunológico. Por exemplo, há evidências de que o exercício a $5^{\circ} \mathrm{C}$ pode culminar em maior comprometimento do que o exercício à temperatura mornal (Lavoy et al., 2011). Portanto, condições ambientais podem perturbar a resposta imunológica em atletas. 
O estresse do exercício, o status de hidratação e as condições ambientais são fatores conhecidos por alterarem a resposta imunológica em atletas. Embora a literatura apresente sob forma de revisão sistemática e metanálise os aspectos relacionados ao treinamento físico em jovens atletas (Harries et al., 2012), Estes não indicaram a relação entre a resposta imune e o exercício em jovens atletas.

Neste protocolo, os atletas correram 3.000 metros de corrida e em ambiente não controlado. Até onde sabemos, um estudo investigou um grande grupo de indivíduos divididos em dois grupos: 1 grupo: correr ou esquiar pelo menos 1 hora sem pausas; 2 grupo) bicicleta por $5 \mathrm{~min}$ em alta resistência e alta rotação. Verificou-se que não há diferença entre os sexos na faixa etária de 19 a 44 anos (Sand et al., 2013). Mas nenhum estudo investigou menores de 18 anos. Portanto, investigamos o sistema imune, resposta hematológica e o estado de hidratação em menores de 18 anos em corridas de 3000 metros para ambos os sexos.

\section{MÉTODOS}

\subsection{PARTICIPANTES}

Neste estudo foram incluídos os seis melhores tempos, levando-se em conta o sexo, dentre os participantes da corrida de 3000 metros nas Olimpíadas Nacionais do Ensino Médio (menores de 18 anos) no Brasil, principal evento nacional para revelar atletas. Todos os participantes estavam em fase competitiva em sua respectiva periodização de treinamento e compareceram ao mesmo centro de treinamento e nenhuma lesão musculoesquelética foi relatada durante os procedimentos. $O$ anonimato dos participantes foi garantido. Foi realizado o mascaramento dos participantes, investigador e avaliador em relação aos resultados, hipóteses e desfechos analisados.

Para ser incluído, os participantes deveriam apresentar ausência de anemia, inflamação, diabetes, hipertensão, doença cardiovascular e lesões musculoesqueléticas nos seis meses anteriores à coleta de dados. Além disso, foram orientados a abster-se de drogas antiinflamatórias, analgésicas, alimentos 
termogênicos, bebidas alcoólicas e tabaco e a não realizar nenhum exercício não proposto pelo estudo. Todos os indivíduos seguiram uma dieta semelhante e não receberam suplementos especiais.

Os participantes seriam excluídos se não completassem todas as avaliações necessárias.

Declaração de éticaA investigação atendeu ao requisito de pesquisa em seres humanos (Conselho Nacional de Saúde, 2012) aprovado pelo Comitê de Ética e Pesquisa, número 1.064.808 / 2015 da Universidade Federal do Mato Grosso (UFMT).

Design experimentalUma prova de 3.000 metros foi realizada em uma pista oval de 400 metros, com largada para ambos os sexos ao mesmo tempo. A temperatura ambiental foi medida entre $33-35^{\circ} \mathrm{C}$ e umidade de $40 \%$ durante $\mathrm{O}$ estudo, sendo ambos mensurados com termohigrômetro digital. Os treinadores incentivaram os atletas a um esforço máximo durante a prova. Foi utilizado o melhor tempo de finalização abaixo de 18 para cada sexo (masculino: 8 min 26 $\mathrm{s}$; feminino: $10 \mathrm{~min} 24 \mathrm{~s}$ ). Desta forma, foi gerado um estresse semelhante ao de uma competição. A coleta de sangue foi realizada em jejum, pré-corrida, póscorrida e recuperação (60 minutos).

Os atletas poderiam ter água e comida ad libitum antes da corrida. Coleta e análise de sangueAmostras de sangue venoso foram coletadas da veia antecubital e armazenadas a $8^{\circ} \mathrm{C}$ imediatamente após a coleta $\mathrm{A}$ amostra foi utilizada para ensaio hematológico em tubos contendo K3 - EDTA (BD Vacutainer $\AA$, New Jersey, EUA) e as análises foram realizadas no Cell DYN 1400 (Abbot Laboratory, Illinois, EUA). Foi medido o número de leucócitos (total e diferencial), eritrócitos, hemoglobina, hematócrito e plaquetas.O estado de hidratação foi verificado a partir do hematócrito. Os cálculos de comparação entre o desempenho na corrida, apresentados nas tabelas, foram adaptados do modelo matemático de variação percentual onde:

Variação percentual $=\frac{(\text { valor final }- \text { valor inicial })}{} \times 100$

Valor Inicial

\section{As adaptações foram:}

- O aumento foi calculado a partir da fórmula: 
Aumento $=\{($ Pós $\times 100) /$ Rep $\}-100 ;$

- O percentual de recuperação em relação ao tempo repouso a partir da formula:

$\operatorname{Rec}-\operatorname{Rep}=\{(\operatorname{Rec} \times 100) / \operatorname{Rep}\}-100$;

- O percentual de recuperação em relação ao tempo Pré a partir da formula:

- Rec-Pré $=\{(\operatorname{Rec} \times 100) /$ Pré $\}-100$;

- O percentual de recuperação em relação ao tempo Pós a partir da formula Rec-Pós $=\{(\operatorname{Rec} \times 100) /$ Pós $\}-100$.

\section{Análise estatística}

Os dados em percentagem foram analisados por ANOVA de duas vias de repetição. Todos os procedimentos de comparação múltipla emparelhados pelo método de Holm-Sidak usando comparações para fator: tempo e analito. Os resultados presentes em tabelas e rótulos são apresentados como média \pm erro padrão da média (EP). O coeficiente de correlação de Pearson foi calculado entre leucócitos e plaquetas. Os resultados foram considerados estatisticamente significativos com $p<0,05$.

\section{RESULTS}

Tabela 1. Características e tempo de chegada na prova de corrida. Valores em média \pm SE.

\begin{tabular}{|c|c|c|c|c|}
\hline \multirow{3}{*}{$\begin{array}{c}\text { Gênero } \\
\text { Homens } \\
(n=3)\end{array}$} & \multicolumn{2}{|c|}{ Antropometia } & \multirow{2}{*}{$\begin{array}{c}\begin{array}{c}\text { Tempo de } \\
\text { cheqada }\end{array} \\
08: 45: 59 \\
\end{array}$} & \multirow[t]{2}{*}{$\begin{array}{l}\text { Tempo de } \\
\text { referência }\end{array}$} \\
\hline & Idade (anos) & $17.7 \pm 2.8$ & & \\
\hline & Estatura $(\mathrm{cm})$ & $176.2 \pm 7.0$ & $08: 50: 22$ & 08:26:00 \\
\hline \multirow{4}{*}{$\begin{array}{l}\text { Mulheres } \\
\qquad(\mathrm{n}=3)\end{array}$} & Massa $(\mathrm{Kg})$ & $62.2 \pm 1.4$ & 09:02:24 & \\
\hline & Idade (anos) & $\underline{16.0 \pm 1.0}$ & $10: 45: 50$ & \multirow{3}{*}{$10: 24: 00$} \\
\hline & Estatura $(\mathrm{cm})$ & $163.0 \pm 2.6$ & $10: 54: 25$ & \\
\hline & Massa $(\mathrm{Kg})$ & $50.0 \pm 3.0$ & $11: 15: 15$ & \\
\hline
\end{tabular}

\section{$\mathrm{SE}=$ standard error}

As características antropométricas dos participantes estãoapresentadas na Tabela 1.

Em nosso resultado, pôde-se perceber que os eritrócitos (masculino: 13\%; feminino; 5\%), hemoglobina (masculino: $9 \%$; feminino: $7 \%$ ) e hematócrito 


\section{5.}

(masculino: 10\%; feminino: 6\%) aumentaram significativamente pós-corrida para ambos os gêneros. Durante o período de recuperação, foi mostrada uma mudança significativa do estado de jejum e do tempo da pré-corrida nos eritrócitos e no hematócrito em atletas do sexo feminino. Além disso, pré-corrida também apresentou mudança significativa na hemoglobina em comparação com o jejum e a recuperação em atletas do sexo feminino (Tabela 2a).

Tabela 2a. Resposta hematológica à prova de 3000 metros. Valores em média \pm SE.

\begin{tabular}{|c|c|c|c|c|c|c|c|c|}
\hline \multirow[b]{2}{*}{ Setor vermelho } & \multicolumn{3}{|c|}{ Homens } & \multirow[b]{2}{*}{$\begin{array}{l}\operatorname{Rec} \\
\text { up }\end{array}$} & & \multicolumn{3}{|c|}{ Mulheres } \\
\hline & $\begin{array}{l}\mathbf{J} \\
\text { ej } \\
\mathbf{u} \\
\mathbf{m}\end{array}$ & $\begin{array}{l}\text { Pr } \\
\text { é- } \\
\text { co } \\
r\end{array}$ & $\begin{array}{l}\text { Pós- } \\
\text { cor }\end{array}$ & & $\begin{array}{c}\text { Jeju } \\
\text { m }\end{array}$ & $\begin{array}{l}\text { Pré } \\
\text { - } \\
\text { cor }\end{array}$ & $\begin{array}{l}\text { Pós } \\
\text {-cor }\end{array}$ & $\begin{array}{l}\text { Re } \\
\text { cu } \\
p\end{array}$ \\
\hline Eritrócitos (1012/L) & $\begin{array}{r}4.6 \pm 0 \\
.2\end{array}$ & $\begin{array}{l}4 . \\
6 \pm \\
0 . \\
1\end{array}$ & $\begin{array}{l}4.9 \pm \\
0.1^{*}\end{array}$ & $\begin{array}{c}4.4 \\
\pm 0 \\
1\end{array}$ & $\begin{array}{c}4.4 \pm \\
0.3\end{array}$ & $\begin{array}{l}4.4 \\
\pm 0 \\
3\end{array}$ & $\begin{array}{l}4.7 \pm \\
0.3^{*}\end{array}$ & $\begin{array}{l}4.3 \pm \\
0.3 \ddagger\end{array}$ \\
\hline $\begin{array}{l}\text { Hemoglobina } \\
(\mathrm{mmol} / \mathrm{L})\end{array}$ & $\begin{array}{r}8.6 \pm 0 \\
.2\end{array}$ & $\begin{array}{l}8 . \\
7 \pm \\
0 . \\
1\end{array}$ & $\begin{array}{l}9.3 \pm \\
0.2^{*}\end{array}$ & $\begin{array}{c}8.4 \\
\pm 0 \\
1\end{array}$ & $\begin{array}{c}7.9 \pm \\
0.1\end{array}$ & $\begin{array}{l}8.2 \pm \\
0.3 \S\end{array}$ & $\begin{array}{l}8.5 \pm \\
0.2^{*}\end{array}$ & $\begin{array}{l}7.8 \\
\pm 0 . \\
2\end{array}$ \\
\hline Hematocrito (\%) & $\begin{array}{r}41.7 \pm \\
1.2\end{array}$ & $\begin{array}{l}41 . \\
3 \pm \\
0.5\end{array}$ & $\begin{array}{c}44.3 \\
\pm 1.0 \\
*\end{array}$ & $\begin{array}{c}39 . \\
8 \pm 0 \\
.2 \\
\end{array}$ & $\begin{array}{l}38 \overline{8.4} \\
\pm 3.0 \\
\end{array}$ & $\frac{38.7}{ \pm 1.1}$ & $\frac{41.3}{ \pm 1.0^{*}}$ & $\begin{array}{r}\frac{37.5}{ \pm 0.7} \\
\pm\end{array}$ \\
\hline
\end{tabular}

* significativamente diferente de Jejum, Pré-corrida, Recuperação. Fêmea: * significativamente diferente de Jejum, Pré-corrida, Recuperação. § significativamente diferente de Jejum, Recuperação. ‡ significativamente diferente de Jejum, Pré-corrida. Os resultados foram considerados estatisticamentesignificativos para $p<0,001$.

Os leucócitos e subconjuntos mostraram padrão semelhante de aumento para ambos os sexos, embora de magnitude diferente. Desta forma, observouse que os leucócitos aumentaram significativamente (masculino: 66\%;feminino: $86 \%)$ no tempo pós-corrida para ambos os gêneros. Além disso, as plaquetas também aumentaram (masculino: em torno de $22 \%$; feminino: em torno de $25 \%$ ) para ambos os gêneros, sem significância. $O$ coeficiente de correlação de Pearson mostrou importante relação nainteração leucócitos- plaquetas (masculino: $r=0,988 ; p=0,012$; feminino: $r=0,999 ; p=0,000886$ ) (Figura 1). 


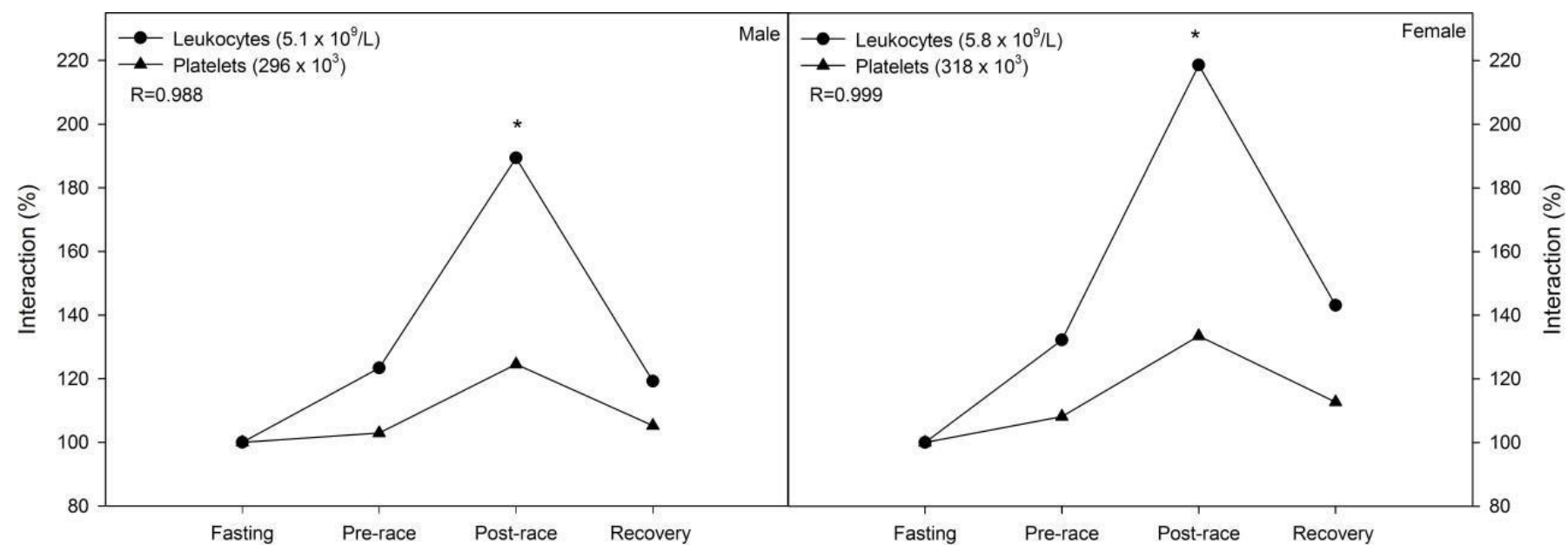

Figura 1. Interações leucócitos-plaquetas. * significativamente diferente de Jejum, Pré-corrida, Recuperação, ambos os sexos. O painel esquerdo é masculino $(R=0,988 ; p=0,012)$ e o painel direito é feminino $(R=0,999 ; p$ $=0,000886)$. Os resultados foram considerados estatisticamente significativos para $p<0,001$. Os valores são mostrados como média \pm SE no rótulo.

Os basófilos aumentaram significativamente (masculino: 267\%; feminino: cerca de 193\%) no pós-corrida. Além disso, os basófilos no tempo précorrida, em comparação com o jejum, aumentaram significantemente (100\%) para o sexo masculino e sem significância, (40\%) em atletas do sexo feminino). Embora, sem significância, os neutrófilos aumentaram similarmente (masculino: quase $36 \%$; feminino: cerca de $33 \%$ ) no pós-corrida. Eosinófilos aumentaram sem significância estatística (sexo masculino: 6,7\%; feminino: aproximadamente $30 \%$ ) no tempo pós-corrida (Figura 2).

Relação entre a Recuperação e os tempos Repouso e Pós.

\begin{tabular}{|c|c|c|c|c|c|c|}
\hline \multirow[b]{2}{*}{ Setor vermelho } & \multicolumn{4}{|c|}{ Rec-Rep } & \multicolumn{2}{|c|}{ Rec-Pós } \\
\hline & Homens & Mulheres & Diferença & Homens & Mulheres & Diferença \\
\hline Eritrócitos (\%) & $-4,35$ & $-2,27$ & $91,63 \%$ & $-10,20$ & $-8,51$ & $85,12 \%$ \\
\hline Hemoglobina (\%) & $-2,32$ & $-1,26$ & $84,13 \%$ & $-9,68$ & $-8,23$ & $17,61 \%$ \\
\hline Hematocrito (\%) & $-4,56$ & $-2,34$ & $94,87 \%$ & $-10,16$ & $-9,20$ & $10,43 \%$ \\
\hline
\end{tabular}

A diferença foi calculada a partir da formula Dif $=\{($ Homens $\times 100) /$ Mulheres $\}-$ 100.

Os linfócitos aumentaram significativamente (masculino: 103\%; feminino: $196 \%$ ) na pós-corrida. Os monócitos tiveram incremento significativo e semelhante (masculino: 106\%; feminino: 107\%) no tempo pós-corrida (Figura 3). 
Tabela 3a. Relação entre os tempos de coleta por gênero para leucócitos e Plaquetas.

\begin{tabular}{|c|c|c|c|c|c|c|c|c|}
\hline \multirow[b]{2}{*}{ Analitos } & \multicolumn{4}{|c|}{ Homens } & \multicolumn{4}{|c|}{ Mulheres } \\
\hline & aumento & Rec-Rep & Rec-Pré & Rec-Pós & aumento & Rec-Rep & Rec-Pré & Rec-Pós \\
\hline Leucócitos (\%) & 90,2 & 17,65 & $-4,8$ & 38,1 & 113,8 & 43,1 & 9,21 & $-33,1$ \\
\hline Plaquetas (\%) & 24,0 & 4,73 & 1,97 & 15,53 & 30,2 & 8,49 & $-1,99$ & $-16,67$ \\
\hline & $\begin{array}{l}\text { foi calcul } \\
\text { do em relac } \\
\text { de recuper } \\
\text { al de recup }\end{array}$ & $\begin{array}{l}\text { lo a partir } \\
\text { o ao tempo } \\
\text { ção em rela } \\
\text { racão em re }\end{array}$ & $\begin{array}{l}\text { formula } \\
\text { pouso a } \\
\text { o ao temp } \\
\text { ão ao ten }\end{array}$ & $\begin{array}{l}\text { nento }=\{(\mathrm{PC} \\
\mathrm{r} \text { da formu } \\
\text { ré a partir } \\
\text { Pós a part }\end{array}$ & $\begin{array}{l}\text { 100) / Rep }\} \\
\text { Rec-Rep }=\{(\operatorname{Re} \\
\text { ormula Rec- } \\
\text { a formula Rec }\end{array}$ & $\begin{array}{l}100 ; \text { O pe } \\
\times 100) / \operatorname{Re} \\
\dot{e}=\{(\operatorname{Rec} \times 1\end{array}$ & $\begin{array}{l}\text { - 100; O } \\
\text { 0) / Pré\} - } \\
\text { 00) / Pós\} }\end{array}$ & \\
\hline
\end{tabular}

Durante a recuperação, os neutrófilos mantiveram o nível sanguíneo, como pode ser observado em ambos os sexos. Outros subgrupos de leucócitos reduziram durante a recuperação em comparação com pós-corrida, principalmente linfócitos (masculino: 154\%; feminino: aproximadamente 248\%), monócitos (masculino: 105\%; feminino: aproximadamente 114\%) e basófilos (masculino: 322\%; feminino: aproximadamente 200\%).

Tabela 2b. Variação dos valores da série vermelha em resposta a corrida de $3000 \mathrm{~m}$ por gênero.

\begin{tabular}{|c|c|c|c|c|c|c|c|c|}
\hline \multirow[b]{2}{*}{ Setor vermelho } & \multicolumn{4}{|c|}{ Homens } & \multicolumn{4}{|c|}{ Mulheres } \\
\hline & Aumento & Rec-Rep & Rec-Pré & Rec-Pós & aumento & Rec-Rep & Rec-Pré & Rec-Pós \\
\hline Eritrócitos (\%) & $+6,52$ & $-4,35$ & $-4,35$ & $-10,20$ & $+6,81$ & $-2,27$ & $-2,27$ & $-8,51$ \\
\hline Hemoglobina (\%) & $+8,14$ & $-2,32$ & $-3,44$ & $-9,68$ & $+7,59$ & $-1,26$ & $-4,88$ & $-8,23$ \\
\hline Hematocrito (\%) & $+6,23$ & $-4,56$ & $-3,63$ & $-10,16$ & $+7,55$ & $-2,34$ & $-3,10$ & $-9,20$ \\
\hline
\end{tabular}

A partir da equação gerada com base no cálculo matemático de variação percentual foi possível observar na tabela $2 \mathrm{~b}$ que o aumento gerado nos eritrócitos foi similar (Meninos $+6,52$; Meninas $+6,81$ ), mas os cálculos de relação entre o tempo de recuperação e os tempos repouso e pré provaindicaram uma recuperação de quase o dobro para meninos em relaçao às meninas (RecRep menino -4,35 e menina -2,27; a Rec-Pré menino -4,35 e menina -2,27).

A tabela 2c traçou uma relação entre a Recuperação e os tempos Repouso e Pós para o setor vermelho do sangue, nestes foi possivel observer uma diferença percentual entre os tempos Rec-Rep entre meninos e meninas de $91,63 \%$ para eritrócitos, $84,13 \%$ para hemoglobin e $94,87 \%$ para o hematócrito. 
Tabela 2c. Relação entre a Recuperação e os tempos Repouso e Pós.

\begin{tabular}{lccccccc}
\hline & \multicolumn{3}{c}{ Rec-Rep } & & \multicolumn{3}{c}{ Rec-Pós } \\
\cline { 2 - 4 } \cline { 7 - 8 } Setor vermelho & Homens & Mulheres & Diferença & & Homens & Mulheres & Diferença \\
\hline Eritrócitos (\%) & $-4,35$ & $-2,27$ & $91,63 \%$ & & $-10,20$ & $-8,51$ & $85,12 \%$ \\
\hline Hemoglobina (\%) & $-2,32$ & $-1,26$ & $84,13 \%$ & & $-9,68$ & $-8,23$ & $17,61 \%$ \\
\hline Hematocrito (\%) & $-4,56$ & $-2,34$ & $94,87 \%$ & & $-10,16$ & $-9,20$ & $10,43 \%$ \\
\hline
\end{tabular}

A diferença foi calculada a partir da formula Dif $=\{($ Homens $\times 100) /$ Mulheres $\}-$ 100.

A partir da tabela 3a foi possível observar a relação entre os tempos de coleta por gênero para leucócitos e Plaquetas. Nesta os aumentos foram similares para os gêneros, mas com relações entre recuperação e repouso e recuperação e tempo pré diferentes. Para os leucócitos os dados Rec-Rep e Rec-Pré de meninos foi menos da metade que o das meninas [(Rec-Repmeninos 17,65 e meninas 43,1)(Rec-Pré meninos -4,8 e meninas 9,21)]. Trombócitos [(Rep-Rep meninos 4,73 e meninas 8,49)(Rec-Pré meninos 1,97 emeninas $1,99)$.

Tabela 3a. Relação entre os tempos de coleta por gênero para leucócitos e Plaquetas.

\begin{tabular}{|c|c|c|c|c|c|c|c|c|}
\hline \multirow[b]{2}{*}{ Analitos } & \multicolumn{3}{|c|}{ Homens } & \multirow[b]{2}{*}{ Rec-Pós } & \multicolumn{4}{|c|}{ Mulheres } \\
\hline & aumento & Rec-Rep & Rec-Pré & & aumento & Rec-Rep & Rec-Pré & Rec-Pós \\
\hline Leucócitos (\%) & 90,2 & 17,65 & $-4,8$ & 38,1 & 113,8 & $\underline{43,1}$ & $\underline{9,21}$ & $-33,1$ \\
\hline Plaquetas (\%) & 24,0 & 4,73 & 1,97 & 15,53 & 30,2 & $\underline{8,49}$ & $-1,99$ & $-16,67$ \\
\hline
\end{tabular}

O aumento foi calculado a partir da formula Aumento $=\{($ Pós $\times 100) /$ Rep $\}-100 ; O$ percentual de recuperação em relação ao tempo repouso a partir da formula $\operatorname{Rec}-\operatorname{Rep}=\{(\operatorname{Rec} \times 100) / \operatorname{Rep}\}$ - 100; O percentual de recuperação em relação ao tempo Pré a partir da formula Rec-Pré= $\{(\operatorname{Rec} \times 100)$ / Pré $\}$ - 100;

A tabela $3 b$ mostrou que a diferença entre os leucócitos do tempo de recuperação entre os sexos foi de $-27,8$.

Tabela 3b. Relação entre os tempos para Leucócitos e Plaquetas em relação ao gênero $\left(10^{3} / \mathrm{mm}^{3}\right)$.

\begin{tabular}{|c|c|c|c|c|c|c|c|c|}
\hline & \multicolumn{4}{|c|}{ Leucócitos } & \multicolumn{4}{|c|}{ Plaquetas } \\
\hline & Rep & Pré & Pós & Rec & Rep & Pré & Pós & Rec \\
\hline Homens & 5,1 & 6,3 & 9,7 & 6,0 & 296 & 304 & 367 & 310 \\
\hline Mulheres & 5,8 & 7,6 & 12,4 & 8,3 & 318 & 352 & 414 & 345 \\
\hline Diferença & $-12,1$ & $-17,1$ & $-21,8$ & $-27,8$ & $-6,9$ & $-13,6$ & $\begin{array}{l}-11,3 \\
\end{array}$ & $-10,1$ \\
\hline
\end{tabular}

A diferença foi calculada a partir da formula Dif $=\{($ Homens $\times 100) /$ Mulheres $\}-100$.

A tabela 4a mostrou uma inversão de comportamento da relaçao RecPós para os eosinófios com -50 para meninos e 38,5 para meninas. E a tabela 
Tabela 4a. Relação entre os tempos de coleta por gênero para Eosinófilos e Basófilos.

\begin{tabular}{|c|c|c|c|c|c|c|c|c|}
\hline \multirow[b]{2}{*}{ Setor branco } & \multicolumn{4}{|c|}{ Homens } & \multicolumn{4}{|c|}{ Mulheres } \\
\hline & aumento & Rec-Rep & Rec-Pré & Rec-Pós & aumento & Rec-Rep & Rec-Pré & Rec-Pós \\
\hline Eosinófilos (\%) & 0 & -50 & $-41,7$ & -50 & 18,2 & $-27,5$ & $-33,3$ & 38,5 \\
\hline Basófilos (\%) & 400 & 50 & -25 & -70 & 250 & 25 & 0 & $-64,3$ \\
\hline
\end{tabular}

O aumento foi calculado a partir da formula Aumento $=\{($ Pós x 100) / Rep $\}-$ 100; O percentual de recuperação em relação ao tempo repouso a partir da formula $\operatorname{Rec}-\operatorname{Rep}=\{(\operatorname{Rec} \times 100) / \operatorname{Rep}\}-100 ;$ O percentual de recuperação em relação ao tempo Pré a partir da formula $\mathbf{R e c}-P r e ́=\{(\operatorname{Rec} \times 100) /$ Pré $\}-100 ; 0$ percentual de recuperação em relação ao tempo Pós a partir da formula RecPós $=\{(\operatorname{Rec} \times 100) /$ Pós $\}-100$.

Já quando observados os linfócitos e monócitos, houve um comportamento antagônico entre essas subfamílas de leucócitos, onde os linfócitos apresentaram aumento de 126 para meninos e 215,1 para meninas e monócitos de 114,7 para meninos e 23,4 para meninas. Isto indica um maior aumento de células de memória em meninas e de fagócitos em meninos.

Tabela 4b. Relação entre os tempos para Eosinófilos e Basófilos em relação ao gênero.

\begin{tabular}{|c|c|c|c|c|c|c|c|c|}
\hline \multirow[b]{2}{*}{ Média } & \multicolumn{4}{|c|}{ Eosinófilos } & \multicolumn{4}{|c|}{ Basófilos } \\
\hline & Rep & Pré & Pós & $\operatorname{Rec}$ & Rep & Pré & Pós & $\operatorname{Rec}$ \\
\hline Homens & 0,14 & 0,12 & 0,14 & 0,07 & 0,02 & 0,04 & 0,1 & 0,03 \\
\hline Mulheres & 0,11 & 0,12 & 0,13 & 0,08 & 0,04 & 0,05 & 0,14 & 0,05 \\
\hline Diferença & 27,3 & 0 & 7,7 & $-12,5$ & -50 & -20 & $-28,6$ & -40 \\
\hline
\end{tabular}

A diferença foi calculada a partir da formula Dif $=\{($ Homens $\times 100) /$ Mulheres $\}-100$.

A tabela 5 a mostrou que a diferença na concentração de monócitos já no repouso era de $-55,8$ em relação aos gêneros.

Tabela 5a. Relação entre os tempos de coleta por gênero para Linfócitos e Monócitos.

\begin{tabular}{|c|c|c|c|c|c|c|c|c|}
\hline \multirow[b]{2}{*}{ Setor branco } & \multicolumn{4}{|c|}{ Homens } & \multicolumn{4}{|c|}{ Mulheres } \\
\hline & $\begin{array}{c}\text { Aument } \\
0\end{array}$ & $\begin{array}{l}\text { Rec- } \\
\text { Rep }\end{array}$ & Rec-Pré & Rec-Pós & $\begin{array}{c}\text { aument } \\
0\end{array}$ & $\begin{array}{l}\text { Rec- } \\
\text { Rep }\end{array}$ & Rec-Pré & Rec-Pós \\
\hline Linfócitos (\%) & 126 & -33 & $-44,6$ & $-70,3$ & 215,1 & $-24,8$ & $-37,0$ & $-76,1$ \\
\hline Monócitos (\%) & 114,7 & 11,8 & -5 & $-47,9$ & 23,4 & $-31,2$ & $-7,0$ & $-44,2$ \\
\hline
\end{tabular}

O aumento foi calculado a partir da formula Aumento $=\{($ Pós x 100) / Rep $\}-100 ; O$

percentual de recuperação em relação ao tempo repouso a partir da formula

$\operatorname{Rec}-\operatorname{Rep}=\{(\operatorname{Rec} \times 100) / \operatorname{Rep}\}-100 ;$ O percentual de recuperação em relação ao tempo Pré a partir da formula Rec-Pré $=\{(\operatorname{Rec} \times 100) /$ Pré $\}-100$; O percentual de recuperação em relação ao tempo Pós a partir da formula $\operatorname{Rec}-\mathbf{P o ́ s}=\{(\operatorname{Rec} \mathrm{X}$ 100) / Pós\} -100. 
Tabela 5b. Relação entre os tempos para Linfócitos e Monócitos em relação ao gênero.

\begin{tabular}{lcccccccccc}
\hline \multirow{2}{*}{ Médias } & \multicolumn{4}{c}{ Linfócitos } & & \multicolumn{5}{c}{ Monócitos } \\
\cline { 2 - 8 } \cline { 7 - 10 } & Rep & Pré & Pós & Rec & & Rep & Pré & Pós & Rec \\
\hline Homens & 2,0 & 2,42 & 4,52 & 1,34 & & 0,34 & 0,4 & 0,73 & 0,38 \\
\hline Mulheres & 1,65 & 1,97 & 5,2 & 1,24 & & 0,77 & 0,57 & 0,95 & 0,53 \\
\hline Diferença & 21,2 & 22,8 & $-13,1$ & 8,1 & & $-55,8$ & $-29,8$ & $-23,1$ & $-28,3$ \\
\hline
\end{tabular}

A diferença foi calculada a partir da formula Dif $=\{($ Homens $\times 100) /$ Mulheres $\}-100$.

Em suma, os homens recuperaram seu estado de hidratação em quase o dobro em relação as mulheres $(\mathrm{H} t \neq 94,87 \%)$ - REC-REP. Em relação ao sistema imune e resposta inflamatória, as mulheres tiveram um aumento relativo de mais que o dobro para leucócitos e não recuperaram estes valores, além de apresentarem um aumento de eosinófilos neste mesmo tempo, enquanto os homens apresentaram diminuição. As mulheres apresentaram um aumento de quase o dobro para linfócitos, enquanto os homens tiveram este comportamento para os fagócitos.

\section{DISCUSSION}

O exercício físico extenuante é um bom modelo para entender as alterações imunológicas semelhantes a muitos estresses clínicos, como trauma, queimaduras e sepse (Pedersen and Ullum, 1994). Foi descrito que as alterações hematológicas dependem da intensidade, prolongamento do exercício e estado nutricional dos atletas. Mas estudos envolvendo jovens atletas não são bem documentados. As características antropométricas mostraram o perfil do corredor de cada sexo neste estudo.

Tem sido demonstrado que os leucócitos são demarginados dos tecidos linfáticos para a circulação sanguínea durante o exercício e outros tipos de estresse. Foi estabelecido que os leucócitos são estimulados por lesões humorais, citocinas e membranas celulares (Gleeson et al., 2006). Os leucócitos podem permanecer elevados até 48 horas após o exercício (Cruz et al., 2016). Como pode ser observado em nosso estudo, os leucócitos aumentaram significativamente no pós-exercício, embora sem recuperação total em atletas 
de cada sexo. Além disso, as plaquetas são classicamente responsáveis pela homeostase sanguínea nos mecanismos de engajamento. A integração entre leucócitos e plaquetas foi descrita em estudo com pacientes com sepse (Liverani et al., 2018). Leucócitos e plaquetas tiveram uma correlação notável em atletas. Essa interação pode ser explicada pelo mecanismo associado à inflamação por duas funções: plug homeostático e para preservar a integridade da vasculatura individualmente. Resumidamente, as plaquetas recrutam leucócitos para locais de inflamação e também preservam a integridade dos vasos sanguíneos inflamados para prevenir o sangramento de locais de infiltração de leucócitos (Ho-Tin-Noe et al., 2018).

Os subgrupos de leucócitos mais abundantes são os neutrófilos, estes podem desempenhar um papel através de espécies reativas de oxigênio ao dano muscular durante o exercício (Kruger and Mooren 2014). Ao contrário, as subfrações de leucócitos menos abundantes são os basófilos, que liberam histamina e heparina. Nos nossos resultados, os basófilos mostraram um aumento significativo no pós - exercício para ambos os sexos. Apesar de não ter sido mensurado histamina e heparina, a basofilia observada durante os 3.000 metros de corrida pode estar ligada às propriedades vasodilatadoras e anticoagulantes, devido ao tempo de exposição a altos níveis de temperatura corporal $\left(>32^{\circ} \mathrm{C}\right.$ ) (Mestre-Alfaro et al., 2012).

Foi demonstrado que os linfócitos etm funções bifásicas, aumentam dependendo da intensidade e duração do exercício, seguido por uma redução (30-60 min) no pós-exercício (Gleeson and Bishop 2005). Ambos os sexos apresentaram incremento significativo no pós-exercício, seguido por uma redução não significativa em nosso estudo. Foi descrito que os linfócitos respondem à inflamação promovida pelo exercício. Há muitos subconjuntos de células $\mathrm{T}$ para produzir citocinas pró e anti-inflamatórias em resposta ao exercício. $O$ exercício extenuante reduz o número de células $T$ do tipo 1 para produzir interferon- $\gamma$, uma citocina pró-inflamatória. As células $T$ tipo 2 e Treg produzem interleucina-4 e interleucina-10, citocinas anti-inflamatórias. O equilíbrio de citocinas pró e anti-inflamatórias pode expor o atleta à infecção viral 
e melhorar a recuperação do exercício (Shaw et al., 2018). Além disso, também observamos a mobilização significativa de monócitos para o sangue no pósexercício devido a danos musculares. Esses monócitos mobilizam o infiltrado no músculo esquelético e se diferenciam dos macrófagos que reparam e regeneram o dano ao músculo esquelético (Peake et al., 2005).

Ambos granulócitos e agranulócitos são influenciados pelo exercício. Há pequena diferença em relação à intensidade de resposta de leucócitos e subgrupos ao exercício devido aos sexos. Embora os cientistas da área incentivem o controle laboratorial externo clássico, isto é, umidade etemperatura, e o controle interno, como a fase menstrual e contraceptivos orais em resposta ao exercício (Gillum et al., 2011).

Muito se discute as alterações nas concentrações plasmáticas do componente celular e acelular do sangue, mas pouco valor é dado a saber se esta alteração tem influência direta do estado de hidratação, com hemoconcentração ou hemodiluição e a diferença entre os gêneros deste comportamento.

Armstrong et al., (2016) já indicaram que apesar de não haver relatos de diferenças na percepção de sede em relação ao sexo, as diferenças encontradas no volume relativo dos fluídos entre os gêneros sugere que profissionais de saúde e organizadores de provas esportivas devem observar estas diferenças para planejar seus eventos.

Estratégias personalizadas de hidratação de acordo com sexo, modalidade esportiva e características ambientais desempenham um papel fundamental na otimização do desempenho e na manutenção da saúde de atletas (Belval et al., 2019).

O presente estudo apresenta achados divergentes aos apresentados por estudos comparando a diferença entre os sexo sem utilizar a citada equação de variação percentual (Ribeiro et al., 2014), o que mostra a importância desta forma de análise e sua sensibilidade. Porém, o presente apresentou achados similares, com diferenças sérias entre os sexos, aos estudos que utilizaram o hematócrito para controle do estado de hidratação (Weitkunat et al., 2012). 
Pacientes com doenças cardíacas apresentam maior proporção de neutrófilos/linfócitos e proporção de plaquetas/linfócitos, proporções estas que são marcadores inflamatórios, e recuperação mais lenta da frequência cardíaca após exercício (Yurtdas et al., 2014). Quando comparados parâmetros como volume e intensidade, exercícios com maior intensidade e intervalados causam maior aumento agudo na família de linfócitos e neutrófilos que métodos contínuos e com alto volume (Mathes et al., 2017)

Em corridas de aventura se observa uma forte leucocitose, marcada por aumento do número de neutrófilos e monócitos circulantes. Contrário a hipótese de imunossupressão induzida pelo exercício, uma resposta proliferativa linfocitária aumentada também é induzida por este tipo de exercício (TossigeGomes et al., 2014).

Esta linfocitose parece ter uma correlação com a alta amonemia induzida pelo exercício de alta intensidade, que quando a amonemia é controlada a linfocitose tem o mesmo comportamento (Gonçalves et al., 2012). Mas os valores dos biomarcadores após o treinamento variam drasticamente dependendo do tipo e intensidade do exercício realizado, o que exige cada vez mais especificidade nas análises (Bessa et al., 2016).

Conhecer o tempo necessário para a atingir o equilíbro estável do sistema imune e o controle do processo inflamatório após exercício em diferentes sexos e idade são essenciais, pois sabe-se que o estresse inflamatório gera um aumento na síntese e ação da hepicidina, que é um hormônio peptidico produzido pelos hepatócitos, que em processos inflamatórios tem sua expressão e ação estimuladas pela Interleucina 6 , induzindo um aumento de captação do ferro pelos macrófagos e diminuição da sua captação a nível intestinal, ocasionando hipoferremia, o que pode gerar anemia e dificuldade de transporte de gases (Nemeth et al., 2004).

A ação aumentada da hepicidina diminui a captação não somente do ferro, mais de outros metais divalentes como o cobre, manganes, zinco e cobaltopela DMT-1 (Proteína Transportadora de Metais Divalentes) na intestino (Groto 2010). Essa dimuição da biodisponibilidade de metais divalentes afeta 
diretamente vias metabólicas como o ciclo do ácido cítrico, diminuindo a síntese de nove dos onze intermediários deste ciclo, interferindo negativamente com a produção de energia e de carreadores de prótons, que são essenciais para a fosforilação oxidativa (Muchowska et al., 2019).

Outro fato marcante em processos inflamatórios é a eosinofilia, que em fenômenos de estresse como exercício ou patologias como a síndrome metabólica tem ação pró inflamatória, podendo este família de leucócitos ser um marcador mais barato de inflamação quando comparados a citocinas e hormônios (Moussa et al., 2019).

$O$ aumento de linfócitos no grupo que teve um maior estresse metabólico (feminino) corrobora com achados anteriores do nosso grupo, onde em estudo avaliando a amonemia de atletas, quanto maiores os valores de amônia, maiores os níveis de linfócitos e quando a amonemia era controlada pela ingestão de arginina, tambem se controlava a linfocitose (Gonçalves et al., 2012). 


\section{CONCLUSIONS}

O exercício induz alteração do estado de hidratação e sistema imune, tendo diferenças em relação ao sexo e idade. Neste estudo foi possível observar que os Meninos tem um menor estresse inflamatório, recuperam o seu estado de hidratação e a resposta inflamatória mais rápido que as meninas após uma corrida de 3000 metros.

Conhecer o comportamento destes fenômenos em relação ao sexo e idade pode fornecer parâmetros para a manutenção da saúde de atletas e praticantes de exercícios, já que a não recuperação desta condição antes de uma nova sessão de exercício pode ocasionar, hipoferremia, anemia, diminuição de imunidade e da produção de energia pelo metabolismo.

\subsection{CONTRIBUIÇÕES DO AUTOR}

LCOG, AMMN, LSM e ELF conceberam e projetaram o estudo. LCOG, AMMN e LSM coletaram os dados. FIB, LSM, LCOG, ACHF, ELF e AMMN analisaram os dados e redigiram o manuscrito. Todos os autores leram e forneceram feedback crítico sobre o manuscrito antes de aprovar.

\subsection{CONFORMIDADE COM OS PADRÕES ÉTICOS}

Conflito de interesses

Os autores declaram não haver conflito de interesses.

Aprovação ética

Todos os procedimentos realizados em estudos envolvendo participantes humanos estavam de acordo com os padrões éticos do comitê de ética em pesquisa da Escola de Esportes da Universidade de Leeds Beckett e com a declaração de Helsinque de 1964 e suas emendas posteriores ou padrões éticos comparáveis. 


\section{REFERÊNCIAS}

Aristotelous P, Aphamis G, Sakkas GK, Andreou E, Pantzaris M, Kyprianou T, Hadjigeorgiou GM, Manconi M, Giannaki CD (2018) Effects of controlled dehydration on sleep quality and quantity: A polysomnographic study in healthy young adults. J Res e. 12662: 1-7

Armstrong LE, Johnson EC, Mckenzie AL, Ellis LA, Williamson KH (2016) Endurance Cyclist Fluid Intake, Hydration Status, Thirst, and Thermal Sensations: Gender Differences. Int J Sport Nutr Exerc Metab 26: 161-167

Ayotte D, Corcoran MP (2018) Individualized hydration plans improve performance outcomes for collegiate athletes engaging in in-season training. $J$ Int Soc Sports Nutr 15(27):1-10

Belval LN, Hosokawa Y, Casa DJ, Adams WM, Armstrong LE, Baker LB, Burke L, Cheuvront S, Chiampas J, Gonzales-Alonso J, Huggins RA, Kavouras SA, Lee EC, Mcdermott BP, Miller K, Schlader Z, Sims S, Stearns RL, Troyanos C, Wingo $\mathrm{J}$ (2019) Practical hydration solutions for sports. Nutrients 11:1-15

Bessa A, Oliveira VN, Agostini GG, Oliveira RJS, Oliveira ACS, White GE, Wells GD, Teixeira DNS, Espindola FS (2016) Exercise Intensity And Recovery: Biomarkers Of Injury, Inflammation, And Oxidative Stress. J Strength Cond Res 30(2):311-319

Brolinson PG, Elliott D (2007) Exercise and the immune system. Clin Sports Med 26(3):311-319

\section{https://doi.org/10.1016/j.csm.2007.04.011}

Cruz GH, Orellana JN, Taraco AR, Colmenero BR (2016) Leukocyte Populations are Associated with Heart Rate Variability After a Triathlon. J Hum kinet 54:5563

\section{$\underline{10.1515 / \text { hukin-2016-0036 }}$}

Gillum TL, Kuennen MR, Schneider S, Moseley P (2011) review of sex differences in immune function after aerobic exercise. Exerc Immunol rev 17:104121

Gleeson M (2006) Immune system adaptation in elite athletes. Curr opin clin nutr metab care $9(6): 659-665$

10.1097/01.mco.0000247476.02650.18

Gleeson M, Bishop NC (2005) The T cell and NK cell immune response to exercise. Ann Transplant 10(4):43-48 
Gonçalves LCO, Bessa A, Freitas-Dias R, Luzes F, Werneck-de-Castro JPS, Bassini A, Cameron LC (2012) A sportomics strategy to analyze the ability of arginine to modulate both ammonia and lymphocyte levels in blood after highintensity exercise. J Int Soc Sports Nutr 9:30 http://www.jissn.com/content/9/1/30 Groto HZW (2010) Fisiologia e metabolismo do ferro. Rev Bras Hematol Hemoter $32(2): 8-17$

Harries SK, Lubans DR, Callister R (2012) Resistance training to improve power and sports performance in adolescent athletes: a systematic review and metaanalysis. J Sci Med Sport 15(6):532-540

10.1016/j.jsams.2012.02.005

Hermand E, Chabert C, Hue O (2019) Ultra-endurance events in tropical environments and countermeasures to optimize performances and health. Int $\mathrm{J}$ Hyperthermia 36(1):753-760

Ho-Tin-Noe B, Boulaftali Y, Camerer E (2018) Platelets and vascular integrity: how platelets prevent bleeding in inflammation. Blood 131(3):277-288 10.1182/blood-2017-06-742676

Kruger K, Mooren FC (2014) Exercise-induced leukocyte apoptosis. Exerc Immunol Rev 20:117-134

Lacey J, Corbett J, Forni L, Hooper L, Hughes F, Minto G, Moss C, Price S, Whyte G, Woodcock G, Mythen M, Montgomery H (2019) A multidisciplinary consensus on dehydration: definitions, diagnostic methods and clinical implications. Ann Med 51(3-4):232-251

Lavoy EC, McFarlin BK, Simpson RJ (2011) Immune responses to exercising in a cold environment. Wilderness Environ Med 22(4):343-351 10.1016/j.wem.2011.08.005

Leites GT, Cunha GS, Obeid J, Wilk B, Meyer F, Timmons BW (2016) Thermoregulation in Boys and Men Exercising at the Same Heat Production Per Unit Body Mass. Eur J Appl Physiol 116(7): 1411-9 10.1007/s00421-016-3400-4 Liska D, Mah E, Brisbois T, Barrios PL, Baker LB, Spriet LL (2019) Narrative review of hydratation and selected health outcomes in the general population. Nutrients 11(70):1-29

Liverani E, Mondrinos MJ, Sun S, Kunapuli SP, Kilpatrick LE (2018) Role of Protein Kinase C-delta in regulating platelet activation and platelet-leukocyte interaction during sepsis. PloS One 13(4):e0195379 10.1371/journal.pone.0195379

Mairbaurl H (2013) Red blood cells in sports: effects of exercise and training on oxygen supply by red blood cells. Front Physiol 4:332 10.3389/fphys.2013.00332 
Malisova O, Athanasatou A, Pepa A, Husemann M, Domnik K, Braun H, MoraRodriguez R, Ortega JF, Fernandez-Elias VE, Kapsokefalou M (2016) Water intake and hydration indices in healthy European adults: The European Hydration Research Study (EHRS). Nutrients 8:1-12

Martin ISM, Vilar EC, Orozco DAR, Dubourdieu PM, Barato VP, Barrado MR, Valente A, Bentancor F, Hurtado ADM, Garagarza C (2019) Hydration Status: Influence of Exercise and Diet Quality. Am J Lifestyle Med 13(4):414-423

Mathes S, Mester J, Bloch W, Wahl P (2017) Impact of high-intensity and highvolume exercise on short-term perturbations in the circulating fraction of different cell types. J Sports Med Phys Fitness January-February;57(1-2):130-137 10.23736/S0022-4707.16.05860-6

Mestre-Alfaro A, Ferrer MD, Banquells M, Riera J, Drobnic F, Sureda A, Tur JA, Pos A (2012) Body temperature modulates the antioxidant and acute immune responses to exercise. Free Radic Res 46(6):799-808 $\underline{10.3109 / 10715762.2012 .680193}$

Moro-Garcia MA, Fernandez-Garcia B, Echeverria A, Rodriguez-Alonso M, Suarez-Garcia FM, Solano-Jaurrieta JJ, Lopez-Larrea C, Alonso-Arias R (2014) Frequent participation in high volume exercise throughout life is associated with a more differentiated adaptive immune response. Brain Behav Immun 39: 61-74 Moussa K, Gurung P, Adams-Huet B, Devaraj S, Jiadal I (2019) Increased eosinophils in adipose tissue of metabolic syndrome. J diabetes Complications 33 (8): 535-538

Muchowska KB, Varma SJ, Moran J (2019) Synthesis and breakdown of universal metabolic precursors promoted by iron. Nature 569 (7754): 104-107 Muth T, Pritchett R, Pritchett K, Depaepe J, Blank R (2019) Hydration Status and Perception of Fluid Loss in Male and Female University Rugby Union Players. Int J Exerc Sci 12(3):859-870

Nemeth E, Rivera S, Gabayan B, Keller C, Taudorf S, Pedersen BK, Ganz T (2204) IL-6 mediates hipoferremia of inflammation by inducing the synthesis of the iron regulatory hormone hepcidin. J Clin Invest 113 (9): 1271-1276 Nieman DC (2003) Current perspective on exercise immunology. Curr Sports Med Rep 2(5):239-242

Nuccio RP, Barnes KA, Carter JM, Baker LB (2017) Fluid Balance in Team Sport Athletes and the Effect of Hypohydration on Cognitive, Technical, and Physical Performance. Sports Med 47:1951-1982

Peake JM, Neubauer O, Dela Gatta PA, Nosaka K (2017) Muscle damage and inflammation during recovery from exercise. J Apl Physiol 122(3):559-570 10.1152/japplphysiol.00971.2016 
Peake J, Nosaka K, Suzuki K (2005) Characterization of inflammatory responses to eccentric exercise in humans. Exerc Immunol Rev 11:64-85

Pedersen BK, Ullum H (1994) NK cell response to physical activity: possible mechanisms of action. Med Sci Sports Exerc 26(2):140-146 10.1249/00005768199402000-00003

Pyne DB, Guy JH, Edwards AM (2014) Managing heat and immune stress in athletes with evidence-based strategies. Int J Sports Physiol Perform 9(5):744750 10.1123/ijspp.2014-0232

Ribeiro AS, Avelar A, Schoenfeld BJ, Dias RMR, Altimari LR, Cyrino ES (2014) Resistance training promotes increase in intracellular hydration in men and women. Eur J Sport Sci 14(6):578-585

Rodrigues CA, Leites GT, Meyer F (2019) Thermoregulatory and Perceptual Responses of Lean and Obese Fit and Unfit Girls Exercising in the Heat. J Pediatr S0021-7557(18): 30949-5 10.1016/i.jped.2018.12.011

Sand KL, Flatebo T, Andersen MB, Maghazachi AA (2013) Effects of exercise on leukocytosis and blood hemostasis in 800 healthy young females and males. World J Exp Med 3(1):11-20 10.5493/wjem.v3.i1.11

Schwellnus M, Soligard T (2016) How much is too much? (Part 2) International Olympic Committee consensus statement on load in sport and risk of illness. $\mathrm{Br}$ J Sports Med 50(17):1043-1052 10.1136/bjsports-2016-096572

Shaw DM, Merien F, Braakhuis A, Dulson D (2018) T-cells and their cytokine production: The anti-inflammatory and immunosuppressive effects of strenuous exercise. Cytokine 104:136-142 10.1016/j.cyto.2017.10.001

Simpson RJ, Kunz H, Agha N, Graff R (2015) Exercise and the Regulation of Immune Functions. Prog Mol Biol Transl Sci 135:355-380 10.1016/bs.pmbts.2015.08.001

Tossige-Gomes R, Ottone VO, Oliveira PN, Viana DJS, Araújo TL, Gripp FJ, Rocha-Vieira E (2014) Leukocytosis, muscle damage and increased lymphocyte proliferative response after an adventure sprint race. Braz J Med Biol Res 47(6): 492-8 http://dx.doi.org/10.1590/1414-431X20143187

Vadopalas K, Ratkevicius A, Skurvydas A, Sipaviciene S, Brazaitis M (2019) Preexercise rehydration attenuates central fatigability during 2-min maximum voluntary contraction in hyperthermia. Medicina 55(66):1-11 


\section{QMEALTH}

Weitkunat T, Knechtle B, Knechtle P, Rust CA, Rosemann T (2012) Body composition and hydration status changes in male and female open-water swimmers during an ultra-endurance event. J Sports Sci 30(10): 1003-1013

Yurtdas M, Taylali YT, Aladag N, Ozdemir M, Ceylan Y, Gençaslan M, Akbulut T (2014) Heart rate recovery after exercise and its relation with neutrophil-tolymphocyte ratio in patients with cardiac syndrome X. Coron Artery Dis Vol 25(6): 485-92 